\title{
An environment-stable hydrogel with skin-matchable performance for human-machine interface
}

\author{
Yang Gao, Yuanrui Wang, Shan Xia ${ }^{*}$ and Guanghui Gao
}

\begin{abstract}
Ionic hydrogel-based sensors have shined a spotlight on wearable electronics. However, the sensitivity and reliability of hydrogel devices are significantly hampered by the weak adhesion of skin-sensor interface as well as inferior temperature tolerance. Here, inspired by the structure and composition of dermis, a novel skin-attachable and environment-stable hydrogel was designed by integrating collagen into the LiCl-containing chemically cross-linked polyacrylamide hydrogel. The hydrogel exhibited skin-like mechanical properties of low modulus, superior stretchability as well as excellent elasticity. Furthermore, the introduction of collagen endowed the hydrogel with robust and seamless interfaces with diverse materials, including the curved skin. As a result, the hydrogel is capable of serving as a human-machine interface for collecting reliable electrocardiography (ECG) signals and discerning various human motions, with high sensitivity (gauge factor $=10.7$ ), fast response, negligible hysteresis as well as extensive monitoring range. Notably, the hydrogel that can mimick the temperature-tolerant mechanism of most organisms possesses persistent stabilization of adhesive, conductive, sensory and mechanical performances at subzero or ambient conditions. The skin-inspired strategy paves an effective way for the design of multifunctional materials with potential applications in next-generation electronics.
\end{abstract}

Keywords: hydrogel, human-machine interface, skin-matchable, self-adhesive, environment-stable

\section{INTRODUCTION}

Ionic hydrogels have been recognized as revolutionary candidates for wearable electronics based on the merits of stretchability, biocompatibility as well as organism-similar conductive way, which could achieve real-time electromechanical response in a broad sensing range, promoting the potential applications in personal health- care diagnosis and human motion detection [1-7]. Despite the significant breakthroughs, most ionic hydrogelbased sensors still suffer from low sensitivity [8-10]. In addition, currently available hydrogel sensors generally secure the contact with skin by adhesive tapes, resulting in not only complicated operations and uncomfortable wearing experience, but also unstable signal detection due to the large interface resistance [11-13]. The robust selfadhesion of hydrogel is necessary for enabling the sensors to be seamlessly attached to curvilinear skin and move together with the dynamic human body without slippage and delamination, thus ensuring the accurate monitoring with high signal-to-noise ratio $[14,15]$.

Besides, the hydrogel sensors usually struggle to maintain stable properties at subzero or ambient conditions due to the evaporation and freeze of water, severely hindering the reliability and application range [16-18]. Partially or entirely replacing the water with nonvolatile solvent is an effective method for developing environmentally stable electronics, however, significantly compromising the conductivity of ionic hydrogels $[19,20]$. Accordingly, some strategies are urgent to construct on-skin sensors with critical feature combinations, including self-adhesion, moisture retention, freezeresistance, skin-similar mechanical properties, and high sensitivity.

Human skin with super sensitivity to multiple stimuli is mainly composed of collagen, elastic fiber and inorganic salt, in which collagen as a significant component can work together with elastic fibers to form a three-dimensional (3D) network for imparting softness and elasticity to the skin $[21,22]$. In recent years, collagen has attracted numerous concerns in the fields of artificial skin, tissue engineering and wound dressings, due to their water solubility, good biocompatibility and low antigenicity $[23,24]$. In particular, abundant amino acid groups in

Polymeric and Soft Materials Laboratory, School of Chemical Engineering, Advanced Institute of Materials Science, Changchun University of Technology, Changchun 130012, China

* Corresponding authors (emails: xiashan@ccut.edu.cn (Xia S); ghgao@ccut.edu.cn (Gao G)) 
collagen open up the feasibility of generating diverse physical interactions for constructing an adhesive interface. Furthermore, in nature, ions are the basis of biological activities in organisms, contributing to the transmission of neural sensing signals and the suppression of water crystallization in cells [25-27]. It can be anticipated that the introduction of collagen into ionic hydrogel networks through the simulation of human skin would inspire the development of flexible sensors with expectable features such as remarkable sensitivity, skinattachable ability and environmental adaption.

Herein, inspired by skin, a self-adhesive and temperature-tolerant hydrogel was presented for an environmentstable human-machine interface. Collagen was introduced into a network consisting of chemically crosslinked polyacrylamide (PAAm) and lithium chloride $(\mathrm{LiCl})$, which mimicked the structure of dermis for endowing the hydrogel with ideal softness and stretchability desired for wearable sensors. Impressively, the hydrogel demonstrated repeatable adhesiveness on various substrate surfaces for seamless skin-sensor interface benefited from the molecular structure of collagen. Besides, remarkable stability of flexibility, conductivity and adhesion for the hydrogel were achieved as exposed to subzero or room temperature chronically. As a result, the hydrogel presented superior sensitivity toward mechanical deformation, which could be applied as a human-machine interface for monitoring electrocardiogram (ECG) signals and body motions with negligible hysteresis, rapid response, broad sensing range and durability.

\section{EXPERIMENTAL SECTION}

\section{Materials}

Acrylamide (AAm, 99.0\%), LiCl (99\%), $N, N, N^{\prime}, N^{\prime}$-tetramethylethy lenediamine (TMEDA, 99.5\%), $N, N^{\prime}$-methylene bisacrylamide (MBA, 99.0\%) and potassium persulfate (KPS, 99.5\%) were obtained from Shanghai Aladdin (China). Collagen (90\%, from fish) was supplied by Shanghai Macklin (China).

\section{Preparation of PAAm-collagen composite hydrogels}

Briefly, AAm monomer, collagen, $\mathrm{LiCl}$ and $\mathrm{MBA}$ were firstly dissolved in deionized water $(5 \mathrm{~mL})$ and stirred for $1 \mathrm{~h}$. Then, KPS $(0.03 \mathrm{~g})$ and TMEDA $(15 \mu \mathrm{L})$ as initiators were added with stirring for $5 \mathrm{~min}$. Finally, the polymerization proceeded at $45^{\circ} \mathrm{C}$ for $6 \mathrm{~h}$, obtaining the PAAm $_{x}$-collagen ${ }_{y}$ hydrogel, where the $x$ represented the MBA content and $y$ was the mass ratio of AAm and collagen. The weight ratio of AAm monomer and col- lagen was fixed at 4:0, 4:1, 4:2 and 4:3. The molar content of $\mathrm{LiCl}$ was $0.6,1.2,1.8$ and $2.4 \mathrm{~mol} \mathrm{~L}^{-1}$. The mole ratios of MBA/AAm were $0.025 \%, 0.05 \%, 0.075 \%$ and $0.1 \%$, respectively. For comparison, pure PAAm hydrogel without collagen and PAAm-collagen hydrogel without $\mathrm{LiCl}$ were synthesized using the same method. The compositions of PAAm-collagen hydrogels were displayed in Table S1.

\section{Peeling measurement}

The adhesive strength of PAAm-collagen hydrogel (thickness of $6 \mathrm{~mm}$, width of $20 \mathrm{~mm}$ ) for diverse substrates was surveyed through $90^{\circ}$ peeling tests with a CT31000 texture analyzer (made in Brookfield of USA). Silica rubber, aluminum, glass, titanium and polytetrafluoroethylene (PTFE) were selected as typical testing surfaces, and the test speed was maintained at $5 \mathrm{~mm} \mathrm{~s}^{-1}$. Before measurement, to prevent the deformation of hydrogel in peeling direction, the upper surface of hydrogel was bonded with polyethylene terephthalate film. At least five samples for each component were tested for determining the final bonding strength. Unless otherwise specified, the $\mathrm{LiCl}$ concentration of PAAm-collagen hydrogel in the peeling test was $1.8 \mathrm{~mol} \mathrm{~L}^{-1}$.

\section{Mechanic contact test}

Mechanic contact test was conducted on the PAAmcollagen hydrogel to investigate the adhesive property by the texture analyzer (CT3-1000, made by Brookfield in USA) with a Spherical Nylon probe of $25.44 \mathrm{~mm}$ in diameter. The loading force, dwell time and test velocity were preset as $100 \mathrm{mN}, 60 \mathrm{~s}$, and $0.5 \mathrm{~mm} \mathrm{~s}^{-1}$, respectively. The adhesion energy $\left(W_{\text {adh }}\right)$ of hydrogel could be obtained using the formula below:

$W_{\text {adh }}=\frac{\int F \mathrm{~d} x}{A_{\max }}$,

where $F, x$, and $A_{\max }$ represent the force, displacement and maximum contact area, respectively. $A_{\max }$ is defined as $A_{\max }=2 \pi r h$, in which $r$ is the representative of radii for probe and $h$ is the distance at the maximum compressive force. Unless otherwise specified, the $\mathrm{LiCl}$ concentration of PAAm-collagen hydrogel in the mechanic contact test was $1.8 \mathrm{~mol} \mathrm{~L}^{-1}$.

\section{Mechanical measurement}

The tensile measurements of PAAm-collagen hydrogels were executed by a universal testing machine with a load of $100 \mathrm{~N}$ (AGX-X, SHIMADZU, Japan). Dumbbellshaped hydrogel samples of $4 \mathrm{~mm}$ width and $3 \mathrm{~mm}$ 
thickness were measured with a constant speed $\left(100 \mathrm{~mm} \mathrm{~min}^{-1}\right)$. The elastic modulus and toughness were obtained by calculating the slope at $0 \%-20 \%$ strain and integral area of stress-strain curves, respectively. For the cyclic loading-unloading tests, the hydrogels were stretched to the preset strain and the dissipated energy was obtained from the integral area of tension-relaxation curves. Unless otherwise specified, the $\mathrm{LiCl}$ concentration of PAAm-collagen hydrogel in mechanical measurement was $1.8 \mathrm{~mol} \mathrm{~L}^{-1}$.

\section{Rheological measurement}

The rheological measurements were performed on the PAAm $_{0.075}$-collagen $_{4: 1}$ hydrogel with $1.8 \mathrm{~mol} \mathrm{~L}^{-1} \mathrm{LiCl}$ by Modular Compact Rheometer of MCR 302 (Anton Paar) with parallel plate in $25 \mathrm{~mm}$ diameter. The shear strain tests ranging from $0.01 \%$ to $1000 \%$ were carried out with a stationary frequency of $10 \mathrm{rad} \mathrm{s}^{-1}$. The frequency sweep measurements $\left(0.1-100 \mathrm{rad} \mathrm{s}^{-1}\right)$ were implemented at the strain of $0.1 \%$. Temperature sweep tests were used to determine the freeze tolerance of $\mathrm{PAAm}_{0.075}$-collagen $_{4: 1}$ hydrogel with $2.4 \mathrm{~mol} \mathrm{~L}^{-1} \mathrm{LiCl}$. The temperature was changed from 50 to $-20^{\circ} \mathrm{C}$ with a cooling rate of $5^{\circ} \mathrm{C} \min ^{-1}$.

\section{Electrical measurement}

The conductive performance of PAAm-collagen hydrogel was measured through the four-probe AC impedance spectrum on an electrochemical workstation (PGSTAT302N, Metrohm). The conductivity $\left(\sigma, \mathrm{S} \mathrm{cm}^{-1}\right)$ of hydrogels was defined as

$\sigma=D /(R \times S)$,

where $D(\mathrm{~cm})$ is the spacing distance between every two probes, $R(\Omega)$ is the expression of resistance of hydrogel, $S\left(\mathrm{~cm}^{2}\right)$ refers to the area of hydrogel cross-section.

The resistance changes of PAAm ${ }_{0.075}$-collagen $_{4: 1}$ hydrogel with $1.8 \mathrm{~mol} \mathrm{~L}^{-1} \mathrm{LiCl}$ under strain or pressure were recorded by the tensile machine associated with B35T digital multimeter (OWON, China). Besides, the hydrogel was applied as a wearable sensor for the real-time detection of whole-body movements through connecting with a digital multimeter by wires. The relative resistance variations of hydrogel were obtained by the equation below:

$\Delta R / R_{0}=\left(R-R_{0}\right) / R_{0} \times 100 \%$,

where $R_{0}$ and $R$ are the initial resistance and resistance after deformation.

The gauge factor (GF), which could indicate the sensitivity of the hydrogel sensor to applied strain $(\varepsilon)$, was acquired by the formula as follows: $\mathrm{GF}=\left[\Delta R / R_{0}\right] / \varepsilon$.

The electrocardiography (ECG) signals were recorded by an Easy ECG Monitor (Prince 180B, Heal Force) through using the adhesive PAAm-collagen hydrogel as electrodes. The signals were transmitted to the smartphone in real-time via Bluetooth.

\section{DSC measurement}

The differential scanning calorimetry (DSC) using DSC-1 (Mettler Toledo, USA) was devoted to evaluating the antiicing ability of PAAm $_{0.075}$-collagen $_{4: 1}$ hydrogel with different concentrations of $\mathrm{LiCl}$. The testing temperature varied from 10 to $-50^{\circ} \mathrm{C}$ with a constant speed of $5^{\circ} \mathrm{C} \mathrm{min}^{-1}$.

\section{Water retention test}

The PAAm ${ }_{0.075}$-collagen $_{4: 1}$ hydrogels with varied concentrations of $\mathrm{LiCl}$ were placed at an oven with a constant temperature of $25^{\circ} \mathrm{C}$ to investigate the anti-drying capacity. The residual mass and resistance change of hydrogels were recorded at regular intervals. The residual mass was obtained from the following equation:

Residual mass $(\%)=W_{t} / W_{0} \times 100 \%$,

where $W_{t}$ and $W_{0}$ are the weight of hydrogel after and before measurement.

\section{Termogravimetric analysis}

The thermogravimetric analysis (TGA) was performed on Q500 (TA, USA) in a nitrogen environment to analyze the moisture retention performance of $\mathrm{PAAm}_{0.075}$ - $\mathrm{Col}$ lagen $_{4: 1}$ hydrogel. The temperature increased from 30 to $70^{\circ} \mathrm{C}$ with a heating rate of $5^{\circ} \mathrm{C} \mathrm{min}^{-1}$.

\section{RESULTS AND DISCUSSION}

In present work, a self-adhesive and temperature-tolerant hydrogel was fabricated by a one-step polymerization of AAm monomer in the presence of collagen and lithium chloride with the MBA as chemical cross-linkers, as schemed in Fig. 1. Collagen could form inter-molecular hydrogen bonds, thereby constructing a PAAm-collagen hydrogel network similar to the structure of dermis in which collagen and elastin form 3D networks to impart skin with softness and elasticity. The PAAm-collagen hydrogel exhibited surprising self-adhesiveness, excellent stretchability and low modulus under the effect of collagen. In addition, from the inspiration of organisms, $\mathrm{LiCl}$ was introduced to effectively improve the water retention and anti-icing capacity of the hydrogel, overcoming the problem that the traditional hydrogel can 


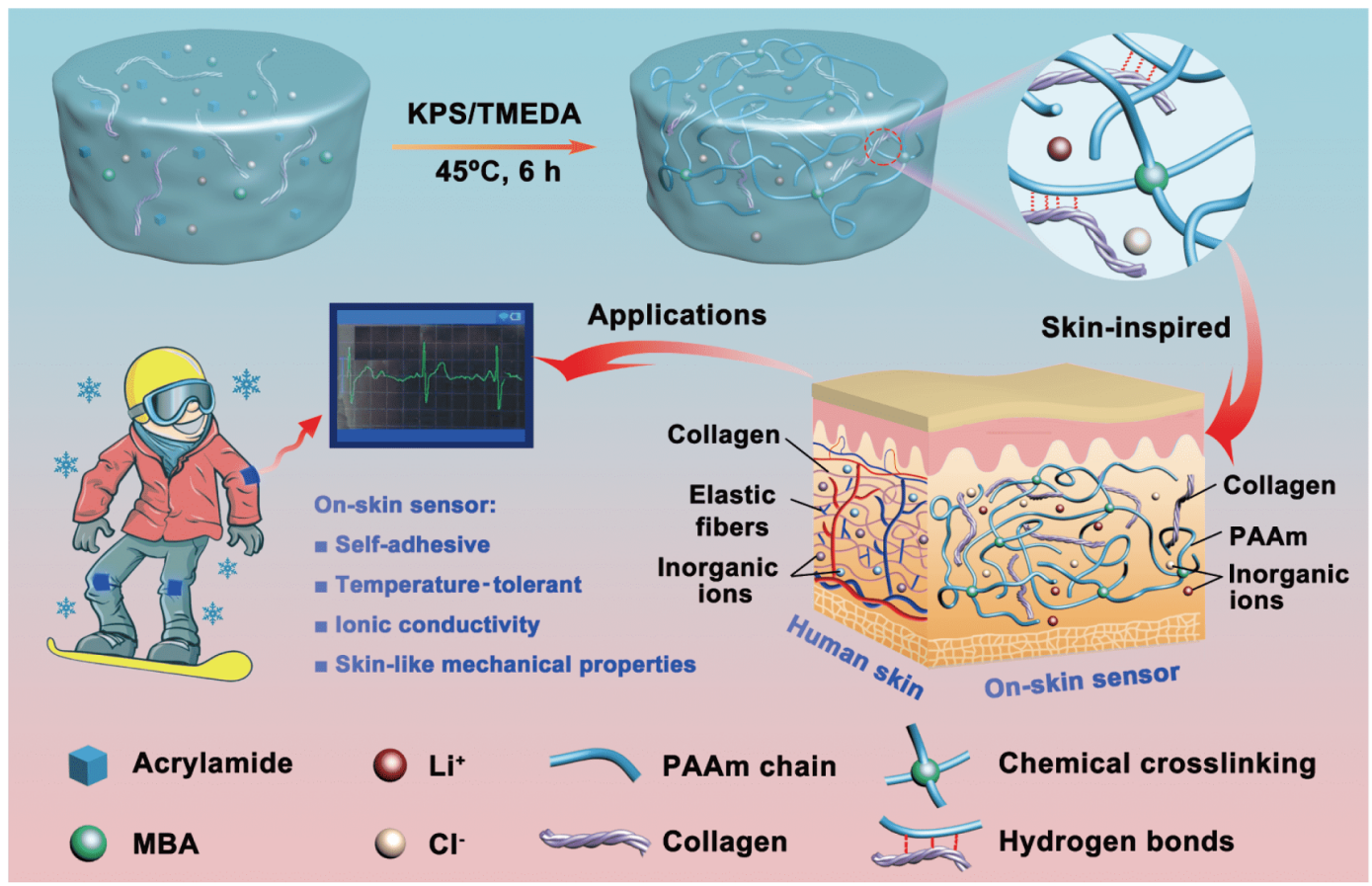

Figure 1 Schematic illustration for preparing skin-inspired PAAm-collagen hydrogel.

hardly maintain stable properties for a long time in cold or normal temperature. The brilliant comprehensive features enabled the PAAm-collagen hydrogel as an ideal human-machine interface for real-time ECG signals recordation and motion detection.

Benefitting from the massive amine acid groups (carboxyl, amino and hydroxyl groups, etc.) in collagen, the obtained PAAm-collagen hydrogel exhibited unique selfadhesive characteristic. As depicted in Fig. 2a, the PAAmcollagen hydrogel adhered to latex glove and finger could be stretched without detachment. Fig. $2 \mathrm{~b}$ further presents that the PAAm-collagen hydrogel could be attached onto a human skin with excellent conformability and good adaptability, which is vital for providing conformal contact and stable integration of hydrogel-skin interface to pledge the motion-sensing accuracy. A series of highly sensitive JKR mechanical contact tests were performed to assess the adhesive performances of the hydrogel assisted by collagen. The force-displacement curves of hydrogels were plotted in Fig. 2c. The PAAm-collagen hydrogel showed a significantly enhanced adhesion force $(280 \mathrm{mN})$ compared with the PAAm hydrogel, attributing to the multiple physical interactions with materials induced by collagen. Furthermore, the contribution of various PAAm/collagen mass ratios on the adhesion was also tested with the same contact force $(0.1 \mathrm{~N})$. As displayed in
Fig. 2d-e, the adhesion force and adhesion energy of the hydrogels gradually increased as the collagen content increased, indicating that the addition of collagen significantly improved the adhesive capability of hydrogels. To further estimate the adhesive behaviors of PAAmcollagen hydrogel, $90^{\circ}$ peeling tests were performed to measure the adhesion on an aluminum alloy substrate (Fig. 2f). The PAAm-collagen hydrogel obtained the maximum adhesive strength $\left(300 \mathrm{~N} \mathrm{~m}^{-1}\right)$ when the mass ratio of AAm and collagen was 4:3, which was 10 times that of pure PAAm $\left(30 \mathrm{~N} \mathrm{~m}^{-1}\right)$. And the effect of MBA content on the adhesive performance of hydrogel was also explored, as shown in Fig. S1. The increase of MBA reduced the peeling strength of hydrogel, which may be ascribed to the destroyed balance between adhesion and cohesion. In addition, the collagen-assisted hydrogel exhibited a universal and robust bonding strength for different substrates. Fig. $2 g$ shows the admirable peel strengths of PAAm-collagen hydrogel on silica rubber, glass, titanium, aluminum and PTFE, respectively. Besides, the PAAm-collagen hydrogel exhibited excellent adhesion behavior without any loss in peel strength during 20 times of repeated peeling tests (Fig. 2h), indicating the durable and reusable adhesion behavior of hydrogels for long-time usage.

Good softness and excellent stretchability are essential 

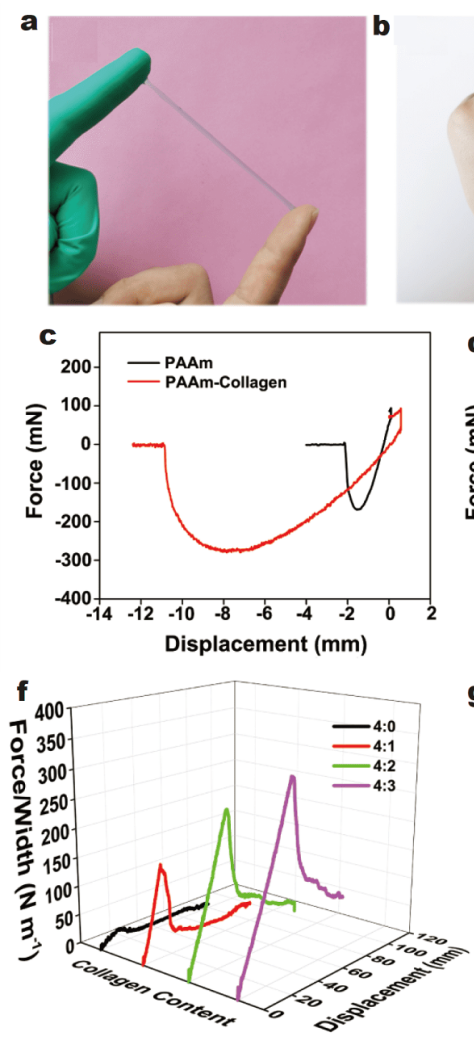
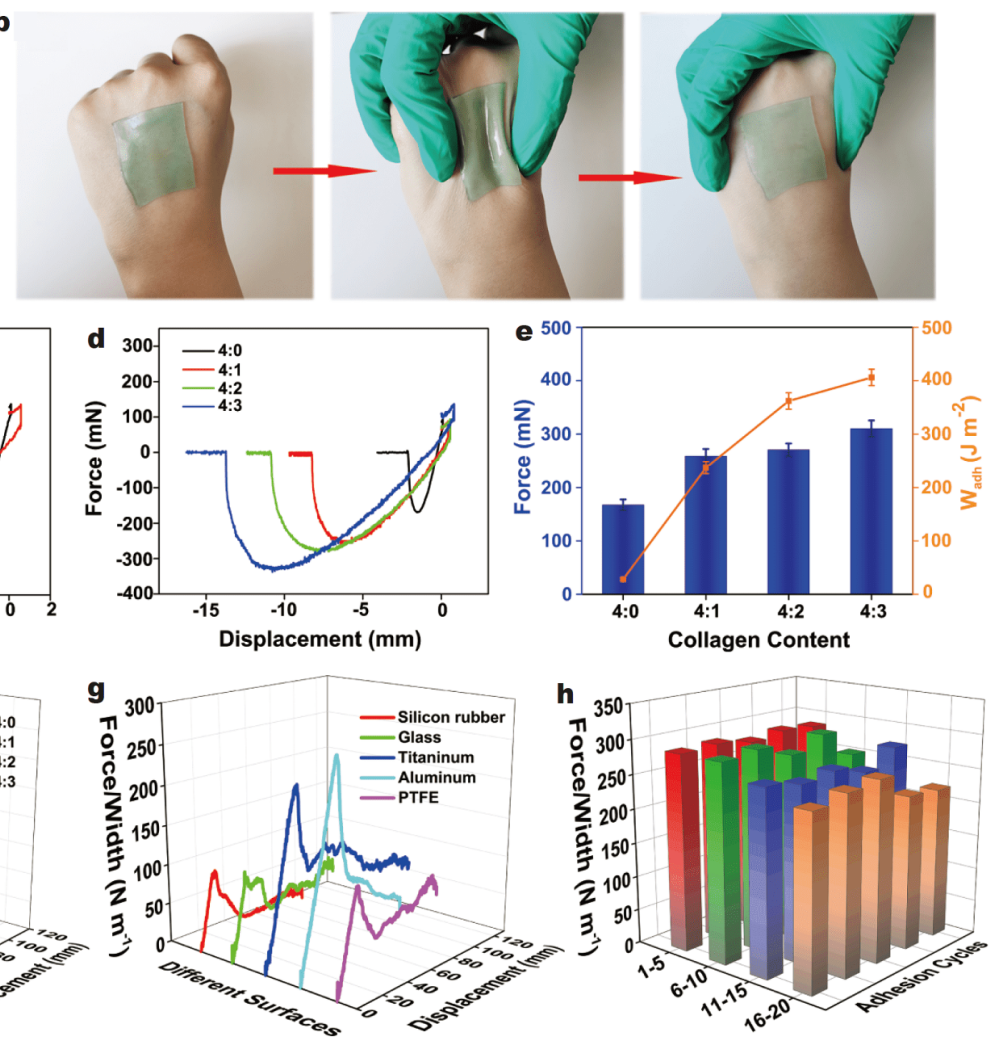

Figure 2 The PAAm ${ }_{0.05}$-collagen $_{4: 2}$ hydrogel adhered to (a) latex gloves and finger with stretchability and (b) the back of hand without interface delamination under squeezing (the hydrogel was dyed by methylene blue). (c) Mechanical contact tests of PAAm $_{0.05}$-collagen $_{4: 2}$ and PAAm P.05 hydrogel. (d) Adhesive force, (e) adhesion energy and (f) $90^{\circ}$ peeling strength of PAAm ${ }_{0.05}$-collagen hydrogel with varied AAm and collagen mass ratios. $90^{\circ}$ Peeling strength of PAAm P. $^{- \text {Collagen }_{4: 2}}(\mathrm{~g})$ on various materials and $(\mathrm{h})$ for 20 times peeling cycles.

features for hydrogel-based sensors to mimic the sensing performance of human skin [28]. According to the dynamic rheology measurement (Fig. S2), the PAAm-collagen hydrogel exhibited a strain and frequencydependent viscoelastic behavior, indicating that the hydrogel possessed the solid elasticity of a viscoelastic material $\left(G^{\prime}>G^{\prime \prime}\right)$. Fig. 3a vividly demonstrates the stretchability, softness and puncture resistance of PAAmcollagen hydrogel. As displayed in Fig. 3b-c, tensile tests were performed on the PAAm-collagen hydrogel to assess the contribution of collagen on mechanical properties. The increase of collagen weakened the tensile strength of hydrogel, but the tensile strain increased step-by-step for well matching with the stretchability of skin (65\%) [12]. The main reason was that collagen interacted with PAAm networks via weak hydrogen bonds and played a role of lubricant in the hydrogel network during stretching [29]. Besides, the PAAm-collagen hydrogel exhibited a low elastic modulus of $10-15 \mathrm{kPa}$, which was comparable to the Young's modulus of human skin, thus ensuring the mechanical compatibility between the hydrogel sensor and human skin as well as improving the wearing comfort $[5,30]$. The mechanical properties of the resultant hydrogel could also be adjusted by varying the content of MBA (Fig. S3). To be specific, the strength and fracture energy of hydrogel tended to increase first and then decrease, while the elastic modulus stepwise increased with the increment of MBA content. Subsequently, a series of tensile cycling measurements were performed. The results in Fig. 3d demonstrate that the hysteresis loop of PAAmcollagen hydrogel increased with varying strain from $100 \%$ to $500 \%$, due to the increased level of destruction in the hydrogel network. Nevertheless, less energy dissipation was exhibited even at the strain of $500 \%$, proving the hydrogel owned excellent resilience. In addition, as shown in Fig. 3e, a large hysteresis loop occurred in the first loading-unloading cycle, which could be attributed to the breakage of physical hydrogen bond cross-links in the network. After the first cycle, the hydrogel showed good fatigue resistance, the dissipated energy was almost 
a
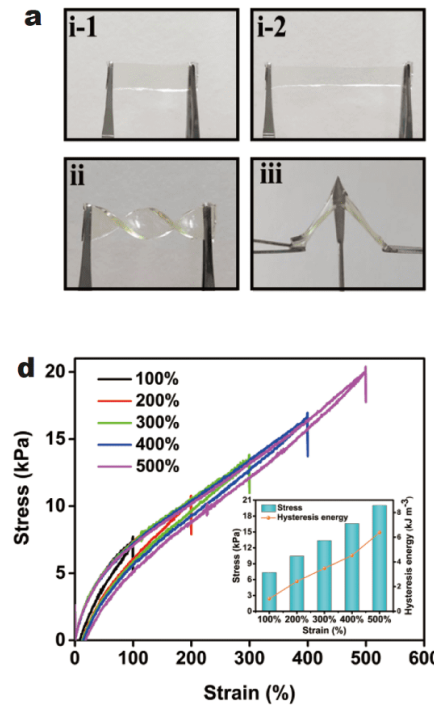
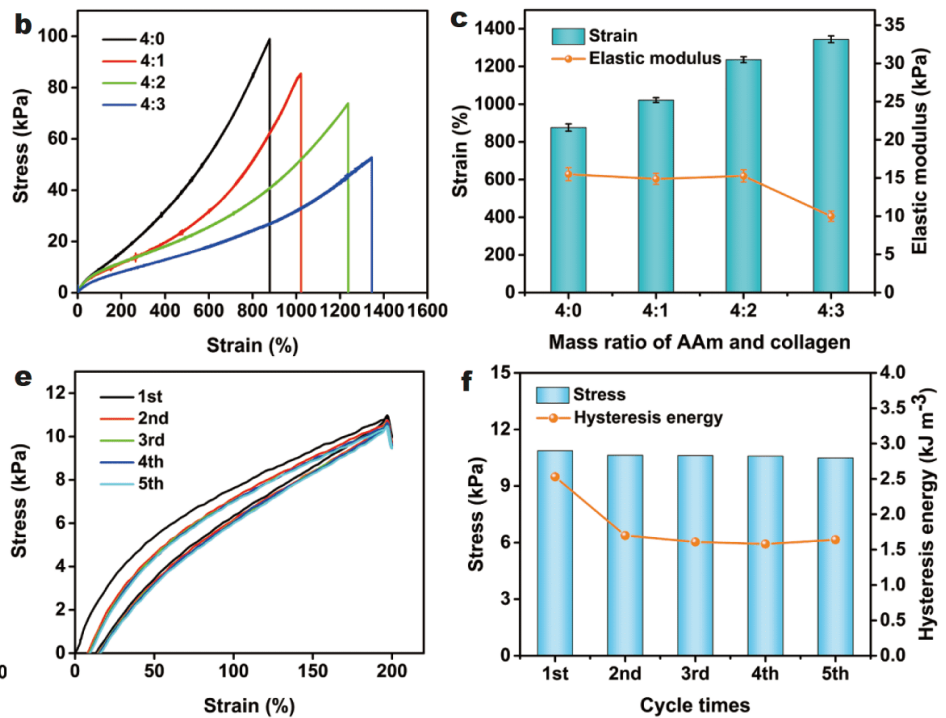

Figure 3 (a) The exhibitions of stretchability, softness and puncture resistance for PAAm-collagen hydrogel. (b) Stress-strain curves of PAAm ${ }_{0.075}$ collagen hydrogel with varied contents of collagen, and (c) corresponding strain and elastic modulus. (d) Cyclic tensile tests of PAAm ${ }_{0.075}{ }^{-{ }^{-}}$ollagen ${ }_{4: 1}$ hydrogel at the strains of $100 \%, 200 \%, 300 \%, 400 \%$ and $500 \%$ (inset: corresponding stress and hysteresis energy). (e) Five loading-unloading cycles of PAAm $_{0.075}$-collagen $_{4: 1}$ hydrogel without dwell time, and (f) corresponding stress and hysteresis energy.

constant during the subsequent consecutive loading-unloading cycles (Fig. 3f). When a hydrogel was connected to the human body as a wearable device, this excellent energy dissipation ability and elastomer-like anti-fatigue could effectively prevent external forces from being transferred to human tissues, thereby protecting the human body from injury. The PAAm-collagen hydrogel could be assembled as a thin layer of on-skin sensor considering the skin-similar mechanical properties. Furthermore, the PAAm-collagen hydrogel demonstated excellent self-adhesion and moderate mechanical properties compared with orther hydrogels in Table S2 [15,3135].

The PAAm-collagen hydrogel possessed excellent conductivity due to the incorporation of $\mathrm{LiCl}$. Here, the hydrogel was applied as an ionic conductor in a circuit for lighting the light-emitting diode (LED) bulb (Fig. 4a). The luminance of LED was weakened by stretching the hydrogel, confirming the increased resistance at larger strain because of the prolonged ion transport channel [36]. It had been proved that the hydrogels were suitable for the application as strain sensors because of the rapid resistance-deformation responsiveness (Fig. 4b). The GF, an indicator of the sensitivity for hydrogel-based sensors, was divided into three stages. The GF was 3.2 in the applied strain range of $0 \%-100 \%$, and then improved gradually to 7.6 in the interval from $100 \%$ to $300 \%$. A high GF of 10.7 was reached at strain above $300 \%$, verifying the superior sensitivity of the PAAm-collagen hydrogel. Fig. $4 c$, $d$ show that the hydrogel sensor exhibited outstanding stability and replicability for the detection of both tiny $(0.25 \%-0.75 \%)$ and relatively large strain (1\%$5 \%)$. As shown in Fig. 4e, the signal intensity was invariable under different tensile speed from 50 to $200 \mathrm{~mm} \mathrm{~min}^{-1}$, signifying that the hydrogel sensor had excellent independence to the test speed. Furthermore, in Fig. 4f, the hydrogel sensor could provide real-time feedback to strain within a short response time $(89 \mathrm{~ms})$ and recovery time $(97 \mathrm{~ms})$. Fig. $4 \mathrm{~g}$ illustrates that the variation of resistance had nearly kept pace with the applied strain without evident sensing delay. Furthermore, 200 tension-relaxation cycles at the strain of $10 \%$ were performed on the hydrogel sensor for investigating the electrical stability (Fig. 4h). Apparently, the relative resistance changes were almost constant after the repeated cycles, highlighting the stability and durability for longterm service life of the hydrogel sensor. As a result, the PAAm-collagen hydrogel exhibited higher sensitivity and quicker response in comparison with previously reported sensors [9,37-44] (Fig. 4i).

Apart from tensile strain, the hydrogels could also be applied as on-skin sensors for monitoring pressure deformation. Fig. $4 \mathrm{j}$ illustrates that the hydrogel sensor was acutely sensitive to a wide pressure range from 2 to 

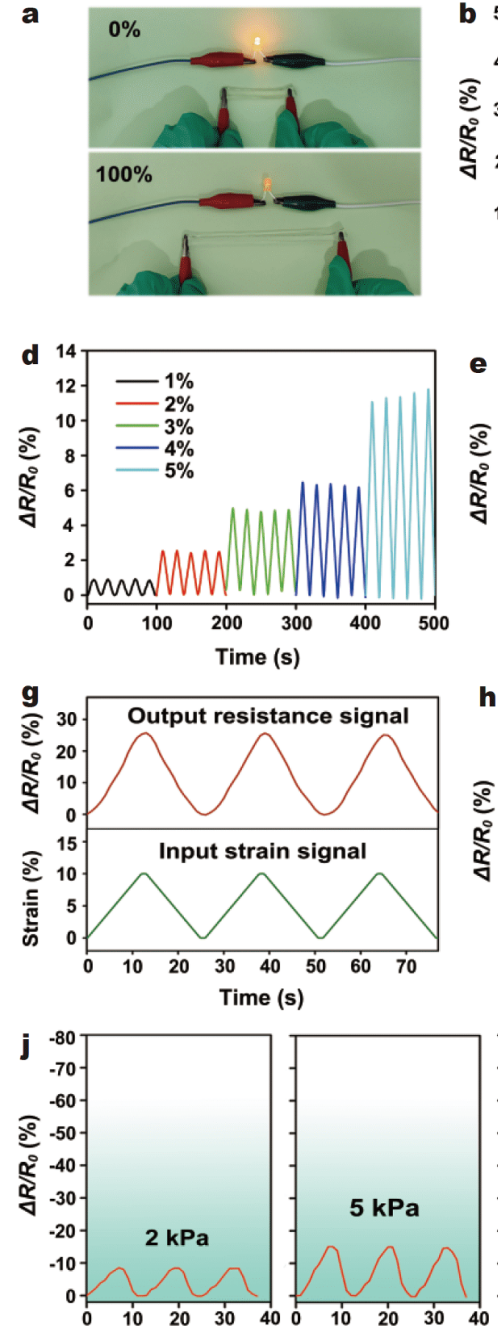

Time (s)

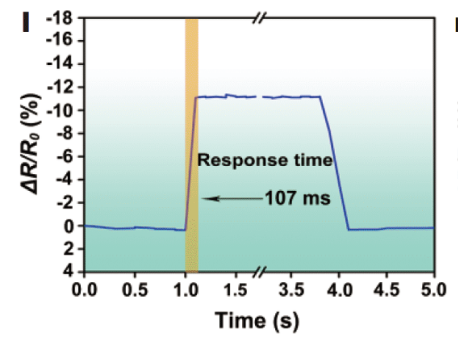

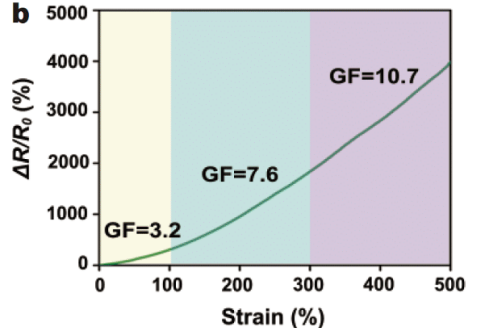
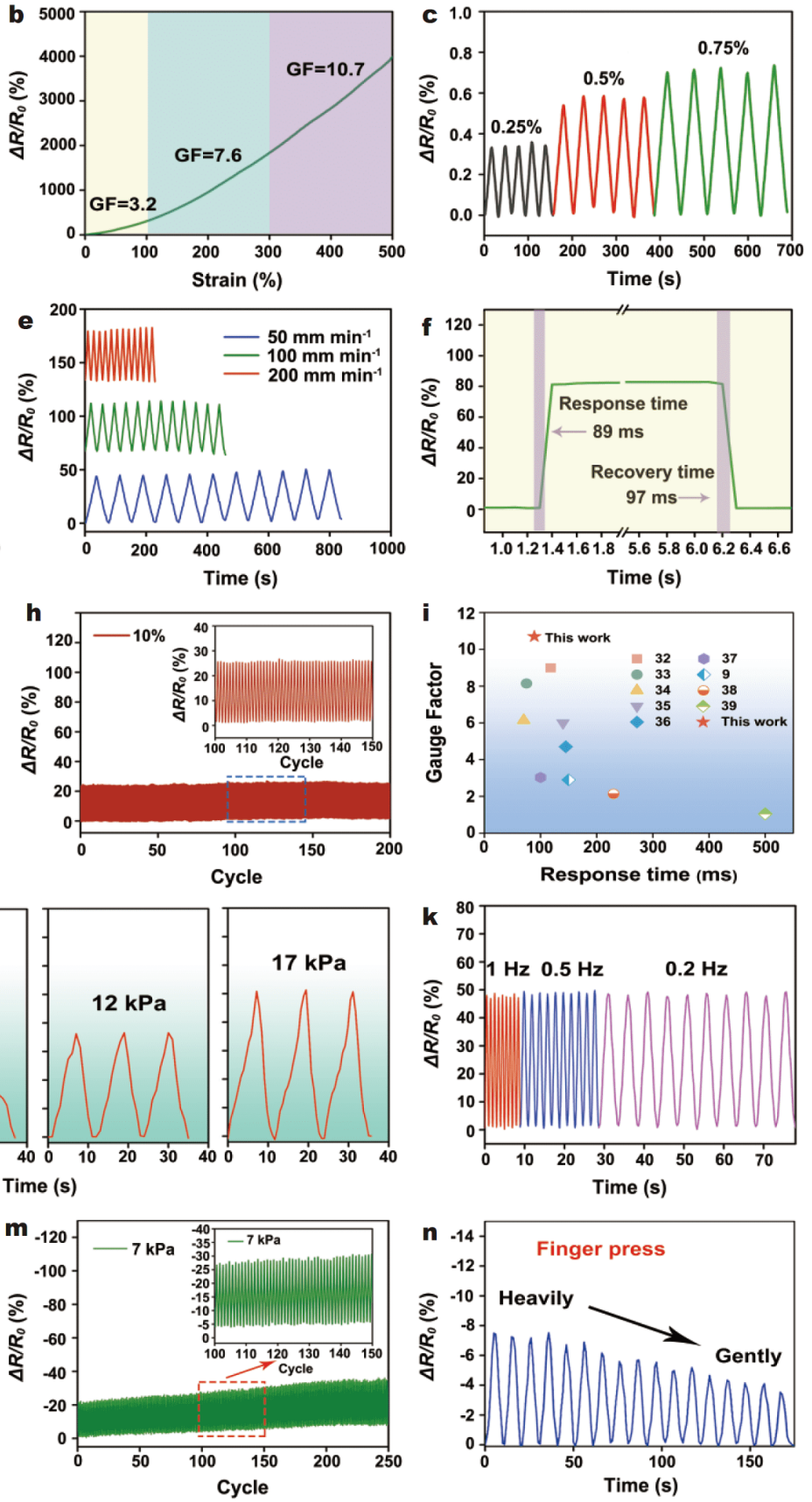

Figure 4 (a) The change of LED brightness with the increase of tensile strain. (b) The variation in relative resistance of hydrogel sensor versus applied strain. $\Delta R / R_{0}$ of the hydrogel sensor under (c) the small-strain range of $0.25 \%-0.75 \%$, (d) the large-strain range of $1 \%-5 \%$, and (e) the tensile speeds of 50-200 $\mathrm{mm} \mathrm{min}^{-1}$. (f) The response and recovery time of the hydrogel sensor. (g) The hysteresis behavior of the hydrogel sensor. (h) $\Delta R / R_{0}$ of the hydrogel sensor at the strain of $10 \%$ for 200 tensile cycles and the enlarged detail of a designated area. (i) Gauge factor and response time of this work compared with other reported sensors. (j) The PAAm-collagen hydrogel as a pressure sensor for monitoring pressure in the range of 2-17 kPa. (k) The variations in relative resistance of the PAAm-collagen hydrogel under different compression frequencies. (l) The response and recovery time of the PAAm-collagen hydrogel pressure sensor. $(\mathrm{m})$ The relative resistance changes of the PAAm-collagen hydrogel at the pressure of $7 \mathrm{kPa}$ for 250 cycles. (n) The $\Delta R / R_{0}$ of the PAAm-collagen hydrogel with the consecutively released pressure from finger.

$17 \mathrm{kPa}$, note that the slight pressure of $2 \mathrm{kPa}$ was also the minimum limit that the skin could perceive [45]. Ob- viously, a higher amplitude of signal waveforms with repeatability was demonstrated by the hydrogel sensor at 
increasing pressure. The hydrogel could also recognize the pressure under different frequencies without the apparent change of $\Delta R / R_{0}$ (Fig. 4k). In addition, the hydrogel sensor could respond to the compressing process in a short time of $107 \mathrm{~ms}$ (Fig. 4l). More importantly, the pressure sensor was sensitive and stable enough to withstand 250 compressive cycles at $7 \mathrm{kPa}$ in Fig. $4 \mathrm{~m}$, illustrating the reliability and endurance of the hydrogel sensor. Furthermore, decreased relative resistance changes were observed with a consecutive decrease in pressure from the finger (Fig. $4 \mathrm{n}$ ).

The stability under the external environment is highly desirable for the practical application of hydrogel sensors, because the water-rich hydrogels are easy to be freezed at subzero temperatures and dehydrated even at ambient conditions, causing the failure of their functions [16]. Freezing tolerance must be implemented by hydrogel sensor for the application in a subzero environment, such as skiing. From the illustration in Fig. 5a, the PAAmcollagen hydrogel without $\mathrm{LiCl}$ turned into white color after being placed at $-20^{\circ} \mathrm{C}$ for $6 \mathrm{~h}$, ascribed to the growth of ice crystals in the hydrogel. However, the LiCl-incorporated hydrogel still remained its initial state, indicating that $\mathrm{LiCl}$ reduced the freezing point of water. DSC test was carried out to investigate the anti-icing ability of the PAAm-collagen hydrogel. Obviously, a stepwise decrease in the crystallization temperature of the hydrogel was achieved by increasing the concentration of $\mathrm{LiCl}$, as a result of the colligative property of ionic compounds [46]. Dynamic temperature-sweep measure-
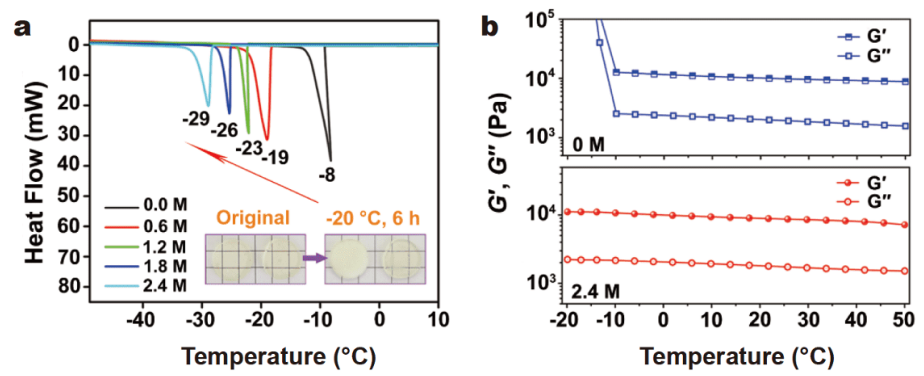

c
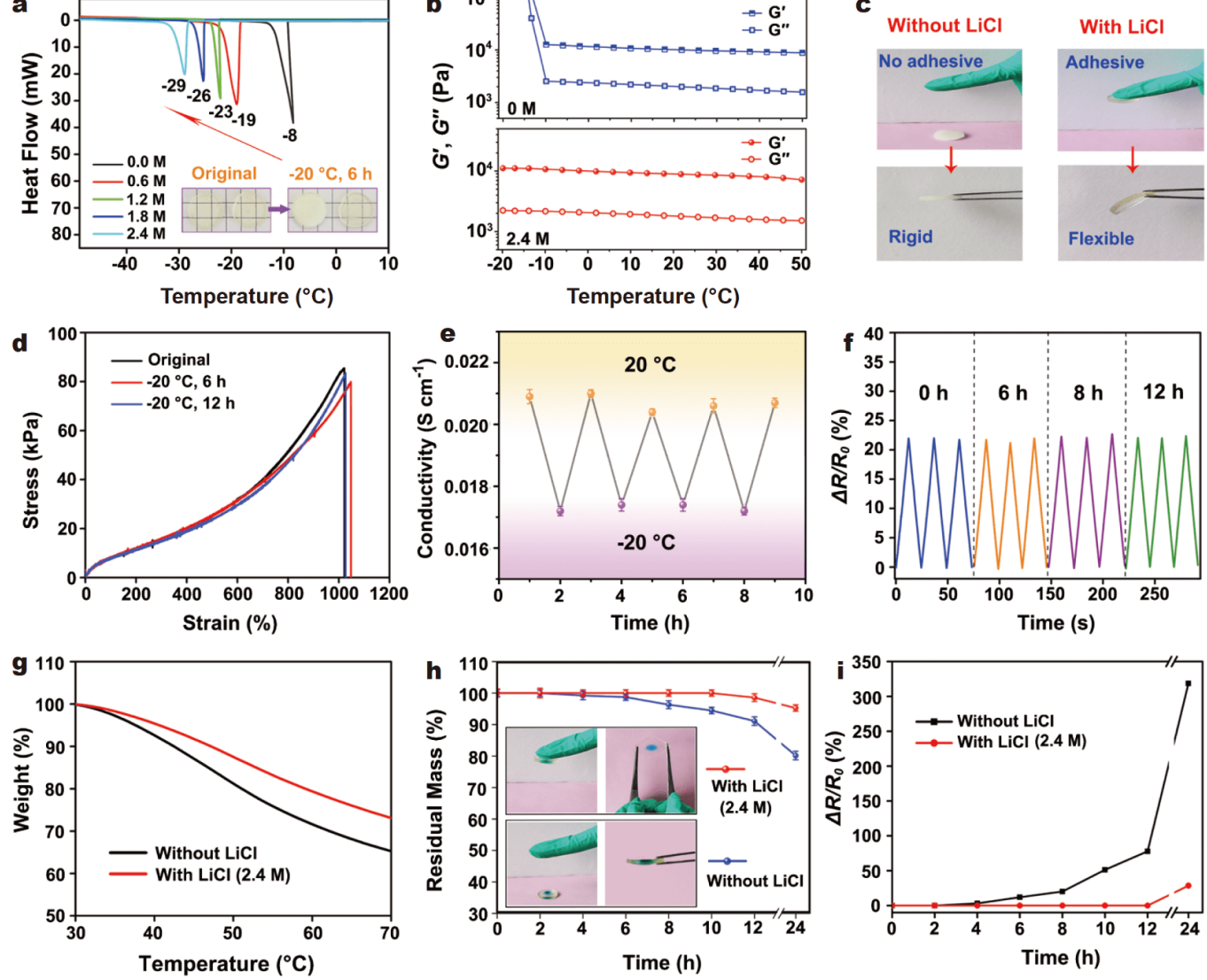

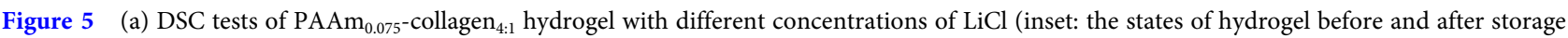
at $-20^{\circ} \mathrm{C}$ for $6 \mathrm{~h}$ ). (b) Temperature sweep measurements of PAAm $\mathrm{P}_{0.075}$ - $^{-}$llagen $_{4: 1}$ hydrogel without and with LiCl. (c) The exhibitions of adhesion and

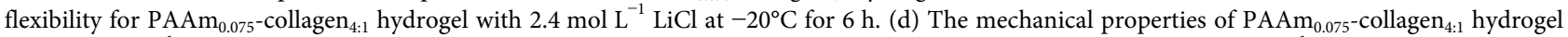
with $2.4 \mathrm{~mol} \mathrm{~L}^{-1} \mathrm{LiCl}$ under $-20^{\circ} \mathrm{C}$ for $6 \mathrm{~h}$ and $12 \mathrm{~h}$. (e) The conductivity of PAAm $\mathrm{P}_{0.075}$-collagen $_{4: 1}$ hydrogel with $2.4 \mathrm{~mol} \mathrm{~L}^{-1} \mathrm{LiCl}$ at $20^{\circ} \mathrm{C}$ and $-20^{\circ} \mathrm{C}$ for 4 cycles. (f) The relative resistance changes of PAAm ${ }_{0.075}$-collagen $_{4: 1}$ hydrogel with $2.4 \mathrm{~mol} \mathrm{~L}^{-1} \mathrm{LiCl}$ at the tensile strain of $10 \%$ after storage at $-20^{\circ} \mathrm{C}$ for $0,6,8$ and $12 \mathrm{~h}$. (g) The TGA curves of PAAm $0.075^{-}$collagen $_{4: 1}$ hydrogel with and without $\mathrm{LiCl}$ in the temperature range of $30-70^{\circ} \mathrm{C}$. (h) The residual mass of $\mathrm{PAAm}_{0.075}$-collagen $_{4: 1}$ hydrogel with and without $\mathrm{LiCl}$ at $25^{\circ} \mathrm{C}$ for $24 \mathrm{~h}$ (inset: the exhibitions of adhesion and flexibility). (i) The resistance change of $\mathrm{PAAm}_{0.075}$-collagen $_{4: 1}$ hydrogel with and without $\mathrm{LiCl}$. 
ment in Fig. 5b also verified the anti-icing behavior and outstanding flexibility of $\mathrm{LiCl}$-assisted hydrogel with the constant moduli in a wide temperature range (50 to $-20^{\circ} \mathrm{C}$ ) [25]. It could be visually observed from Fig. $5 \mathrm{c}$ and Fig. S4 that the LiCl-free PAAm-collagen hydrogel became non-adhesive, rigid, and nonconducting at $-20^{\circ} \mathrm{C}$, while the hydrogel with $\mathrm{LiCl}$ was still adhesive, flexible and conductive invariably. As shown in Fig. 5d, the stretchability and tensile strength of the PAAm-collagen hydrogel with $\mathrm{LiCl}$ at $-20^{\circ} \mathrm{C}$ for varying times almost maintained constant, manifesting the brilliant antifreezing property of the hydrogel. Although the conductivity of the PAAm-collagen hydrogel decreased from $0.021 \mathrm{~S} \mathrm{~cm}^{-1}$ (at $20^{\circ} \mathrm{C}$ ) to $0.017 \mathrm{~S} \mathrm{~cm}^{-1}$ (at $-20^{\circ} \mathrm{C}$ ) due to the constrained ion movement caused by unavoidable freezing of partial water, the hydrogel still exhibited excellent electrical conductivity and cycling stability with negligible deviation at each temperature in Fig. 5e. After the hydrogel was placed at $-20^{\circ} \mathrm{C}$ for different storage times, the relative resistance variations of the hydrogel conductor at the tensile strain of $10 \%$ were recorded in Fig. 5f. The $\Delta R / R_{0}$ of the hydrogel always kept the constant values, indicating that the hydrogel possessed outstanding anti-icing performance. Beyond all that, as shown in Fig. S5, the PAAm-collagen hydrogel frozen at $-20^{\circ} \mathrm{C}$ for $24 \mathrm{~h}$ could still respond to different tensile strains of $5 \%, 10 \%$ and $20 \%$ accurately.

Apart from the freezing-tolerance, moisture retention is vital for pledging the stability of hydrogel at ambient conditions. The original shape of PAAm-collagen hydrogel could be basically considered to maintain invariable after storage at $25^{\circ} \mathrm{C}$ for $24 \mathrm{~h}$, in comparison with the LiCl-free PAAm-collagen hydrogel with contracted volume (Fig. S6). TGA curves in Fig. $5 \mathrm{~g}$ verified that the weight loss of $\mathrm{LiCl}$-assisted PAAm-collagen hydrogel was significantly lower than that of $\mathrm{LiCl}$-free hydrogel, with the temperature increasing from 30 to $70^{\circ} \mathrm{C}$, indicating the contribution of $\mathrm{LiCl}$ for moisture holding of the hydrogel. The possible reason was that the salt ions $\left(\mathrm{Li}^{+}\right.$or $\mathrm{Cl}^{-}$) could strongly bond with water molecule pairs to suppress the evaporation of water [47]. As shown in Fig. 5h, the residual mass of LiCl-free PAAm-collagen hydrogel was only $80 \%$ after exposure to $25^{\circ} \mathrm{C}$ for $24 \mathrm{~h}$. Conversely, the hydrogel with $\mathrm{LiCl}$ possessed a residual mass of $95 \%$ in the condition of equivalence. Thus, in the illustration, the hydrogel without $\mathrm{LiCl}$ lost adhesion and flexibility, while the hydrogel with $\mathrm{LiCl}$ was always able to adhere to skin and stretch. Amazingly, the hydrogel with $\mathrm{LiCl}$ had a residual mass of $81 \%$ even after being placed at $25^{\circ} \mathrm{C}$ for 7 days (Fig. S7). In contrast, the residual mass of the hydrogel without $\mathrm{LiCl}$ reduced to only $40 \%$. The water-retention capacity of PAAm-collagen hydrogel in comparison with orther hydrogels was shown in Table S3 $[41,48-51]$. Due to the excellent water retention ability, the PAAm-collagen hydrogel still possessed high mechanical property and conductivity after being stored at $25^{\circ} \mathrm{C}$ for 7 days (Fig. S8). Besides, a huge increase in resistance of hydrogel without $\mathrm{LiCl}$ was observed in Fig. $5 \mathrm{i}$ when exposed to $25^{\circ} \mathrm{C}$ for $24 \mathrm{~h}$; however, the resistance change of $\mathrm{LiCl}$-contained hydrogel was lower. Fig. S9 shows the resistance changes of the hydrogel with varying $\mathrm{LiCl}$ concentrations after 7 days, further corroborating the contribution of $\mathrm{LiCl}$ to the water retention ability of PAAm-collagen hydrogel. The impressive antiicing and water-retention ability of PAAm-collagen hydrogel hold great promise for working at a wide range of temperatures. However, the high concentration of $\mathrm{LiCl}$ in hydrogel may cause the dehydration of skin, which is a noticeable problem in the applications. Furthermore, the mechanical property and conductivity of PAAm-collagen hydrogel were influenced by relative humidity due to the hygroscopicity of $\mathrm{LiCl}$, as shown in Fig. S10.

Owing to the excellent mechanical compliance, high conductivity, remarkable self-adhesiveness, superior sensing sensitivity and durability, the PAAm-collagen hydrogel demonstrated excellent prospects as an on-skin sensor for detecting subtle physiological signals. As shown in Fig. S11, the hydrogels with self-adhesiveness and low modulus were installed on the electrodes to enhance the bonding force with skin for collecting the ECG signals, greatly reducing the imperfect contact due to the human activity. It could be seen that the ECG waveforms of a volunteer displayed evident separated and negligible fluctuated P, QRS, and T curves in Fig. 6a, b, supplying valuable medical informatics on cardiovascular problems. In addition, the subtle changes in pulse at the wrist were clearly presented and 79 beats per min in a relaxed state were detected by the hydrogel sensor (Fig. 6c). In Fig. 6d, the physiological breathing signals of volunteer were readily tracked via attaching the hydrogel on the chest, whether regular or rapid respiration modes. The hydrogel sensor also possessed the capacity of sensing throat movement during speech, as depicted in Fig. 6e, the phonation of "hydrogel" and "very good" could be recognized by the sensor attributed to the different syllables. And peaks and valleys in the characteristic curves were similar when each word was repeated three times.

In addition to subtle signals, the hydrogel sensor was sensitive enough for detecting complex motions in realtime. It could be seen from Fig. $6 f$ that a gradual increase 
a
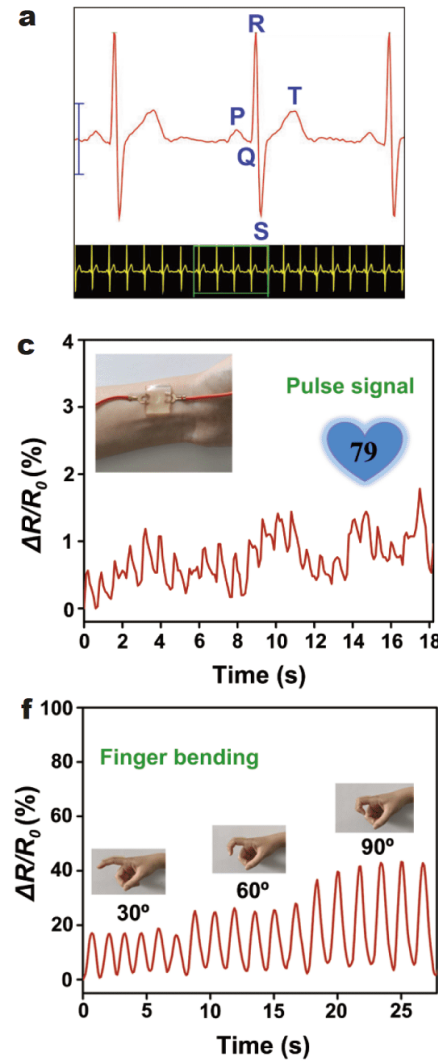

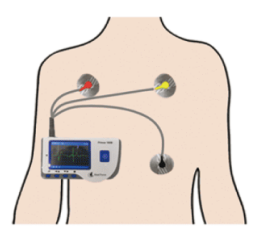

b

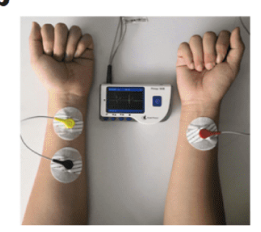

Electrocardiogram (ECG)

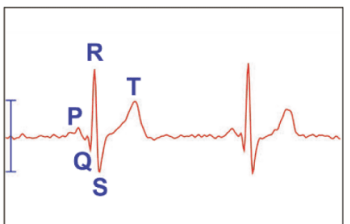

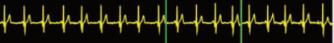
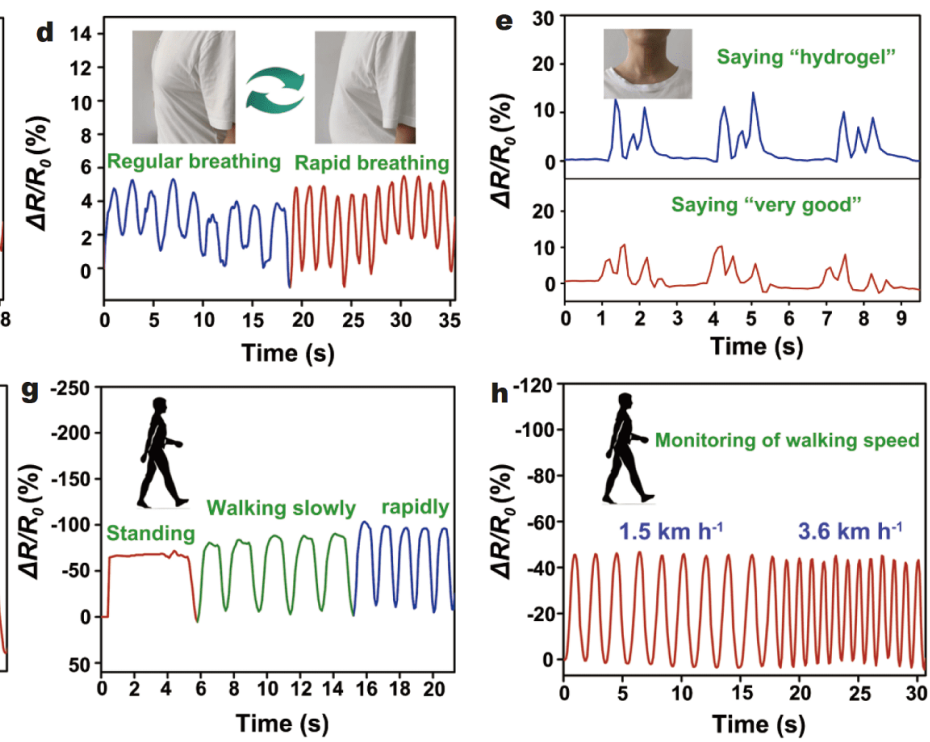

Figure 6 (a) Pairs of hydrogel electrode were attached on the left and right chest of a volunteer with the reference electrode adhered on the left abdomen for detecting ECG signals. (b) Pairs of hydrogel electrodes were attached on the left and right forearms of a volunteer with the reference electrode adhered on left forearm for detecting ECG signals. (c-e) Detecting tiny muscle movements of pulsing, breathing, and speaking. (f) Bending of the finger at different angles $\left(30^{\circ}, 60^{\circ}\right.$ and $\left.90^{\circ}\right)$. (g) Detection of standing and walking with the hydrogel sensor mounted on the sole. (h) Monitoring of the walking speed with the hydrogel sensor.

in $\Delta R / R_{0}$ could be perceived by the sensor when enhancing the bending angles of finger from $30^{\circ}$ to $90^{\circ}$, attributed to the stretching of hydrogel. Moreover, the $\Delta R /$ $R_{0}$ maintained a similar value at the same bending angle, indicating the stability and accuracy of the sensor. Besides, the hydrogel-based sensor was also capable of monitoring the dynamic nodding behavior (Fig. S12). Impressively, the hydrogel could be attached on the cheek directly for the detection of laughing in Fig. S13, showing increased magnitude due to the deformation caused by muscle movement. In addition, the on-skin sensor could sense different exercise states, such as standing and walking, when being mounted on the sole. As shown in Fig. $6 \mathrm{~g}$, the relative resistance was almost constant when the volunteer remained stationary, and rapidly increased under the motion of walking due to the excellent sensitivity. In addition, the hydrogel-based sensor could respond quickly and accurately to walking speed (Fig. 6h), demonstrating a change from sparse to dense in curves with the acceleration of walking from 1.5 to $3.6 \mathrm{~km} \mathrm{~h}^{-1}$.

\section{CONCLUSION}

In summary, inspired by the structure and composition of human skin, a self-adhesive and temperature-tolerant hydrogel was fabricated by chemically crosslinking acrylamide in the presence of collagen and lithium chloride. With the synergistic effect of collagen and PAAm network, the hydrogel exhibited large stretchability, low modulus, elasticity, fatigue resistance as well as robust self-adhesion to diverse material surfaces, which could move with the dynamic skin without delamination. It was worth noting that the hydrogel could maintain stable mechanical properties, conductivity and self-adhesion in a subzero or room temperature for a long time. Furthermore, the hydrogel showed superior sensitivity (GF = 10.7) to mechanical deformation, which was capable of serving as human-machine interface for reliably recording ECG signals and monitoring whole-body movements 
with low hysteresis, broad operating range, fast response and high durability. It is envisioned that the skin-inspired strategy would revolutionize multiple fields, including ionic skin, human-machine interface and wearable sensors.

Received 7 November 2020; accepted 22 January 2021; published online 8 April 2021

1 Li S, Pan H, Wang Y, et al. Polyelectrolyte complex-based selfhealing, fatigue-resistant and anti-freezing hydrogels as highly sensitive ionic skins. J Mater Chem A, 2020, 8: 3667-3675

2 Sun JY, Keplinger C, Whitesides GM, et al. Ionic skin. Adv Mater, 2014, 26: 7608-7614

3 Liu YJ, Cao WT, Ma MG, et al. Ultrasensitive wearable soft strain sensors of conductive, self-healing, and elastic hydrogels with synergistic "soft and hard" hybrid networks. ACS Appl Mater Interfaces, 2017, 9: 25559-25570

4 Ji S, Wan C, Wang T, et al. Water-resistant conformal hybrid electrodes for aquatic endurable electrocardiographic monitoring. Adv Mater, 2020, 32: 2001496

5 Lei Z, Wang Q, Wu P. A multifunctional skin-like sensor based on a 3D printed thermo-responsive hydrogel. Mater Horiz, 2017, 4: 694-700

6 Lei Z, Wu P. A supramolecular biomimetic skin combining a wide spectrum of mechanical properties and multiple sensory capabilities. Nat Commun, 2018, 9: 1134

7 Song J, Chen S, Sun L, et al. Mechanically and electronically robust transparent organohydrogel fibers. Adv Mater, 2020, 32: 1906994

8 Yang B, Yuan W. Highly stretchable and transparent double-network hydrogel ionic conductors as flexible thermal-mechanical dual sensors and electroluminescent devices. ACS Appl Mater Interfaces, 2019, 11: 16765-16775

9 Huang H, Han L, Li J, et al. Super-stretchable, elastic and recoverable ionic conductive hydrogel for wireless wearable, stretchable sensor. J Mater Chem A, 2020, 8: 10291-10300

$10 \mathrm{Gu} \mathrm{G}, \mathrm{Xu} \mathrm{H}$, Peng S, et al. Integrated soft ionotronic skin with stretchable and transparent hydrogel-elastomer ionic sensors for hand-motion monitoring. Soft Robot, 2019, 6: 368-376

11 Sun J, Yuan Y, Lu G, et al. A transparent, stretchable, stable, selfadhesive ionogel-based strain sensor for human motion monitoring. J Mater Chem C, 2019, 7: 11244-11250

12 Song Z, Li W, Bao Y, et al. Breathable and skin-mountable strain sensor with tunable stretchability, sensitivity, and linearity via surface strain delocalization for versatile skin activities' recognition. ACS Appl Mater Interfaces, 2018, 10: 42826-42836

13 Xie $\mathrm{C}$, Wang X, He H, et al. Mussel-inspired hydrogels for selfadhesive bioelectronics. Adv Funct Mater, 2020, 30: 1909954

14 Wang P, Pei D, Wang Z, et al. Biocompatible and self-healing ionic gel skin as shape-adaptable and skin-adhering sensor of human motions. Chem Eng J, 2020, 398: 125540

15 Pei X, Zhang H, Zhou Y, et al. Stretchable, self-healing and tissueadhesive zwitterionic hydrogels as strain sensors for wireless monitoring of organ motions. Mater Horiz, 2020, 7: 1872-1882

16 Han L, Liu K, Wang M, et al. Mussel-inspired adhesive and conductive hydrogel with long-lasting moisture and extreme temperature tolerance. Adv Funct Mater, 2018, 28: 1704195

17 Liao H, Guo X, Wan P, et al. Conductive MXene nanocomposite organohydrogel for flexible, healable, low-temperature tolerant strain sensors. Adv Funct Mater, 2019, 29: 1904507

$18 \mathrm{Wu}$ X, Liao H, Ma D, et al. A wearable, self-adhesive, long-lastingly moist and healable epidermal sensor assembled from conductive mxene nanocomposites. J Mater Chem C, 2020, 8: 1788-1795

19 Chen H, Ren X, Gao G. Skin-inspired gels with toughness, antifreezing, conductivity, and remoldability. ACS Appl Mater Interfaces, 2019, 11: 28336-28344

20 Qin Z, Sun X, Zhang H, et al. A transparent, ultrastretchable and fully recyclable gelatin organohydrogel based electronic sensor with broad operating temperature. J Mater Chem A, 2020, 8: 44474456

21 Zhang X, Sheng N, Wang L, et al. Supramolecular nanofibrillar hydrogels as highly stretchable, elastic and sensitive ionic sensors. Mater Horiz, 2019, 6: 326-333

22 Chen S, Sun L, Zhou X, et al. Mechanically and biologically skinlike elastomers for bio-integrated electronics. Nat Commun, 2020, 11: 1107

23 Shoulders MD, Raines RT. Collagen structure and stability. Annu Rev Biochem, 2010, 78: 929-958

24 Lee CH, Singla A, Lee Y. Biomedical applications of collagen. Int J Pharm, 2001, 221: 1-22

25 Sui X, Guo H, Chen P, et al. Zwitterionic osmolyte-based hydrogels with antifreezing property, high conductivity, and stable flexibility at subzero temperature. Adv Funct Mater, 2019, 30: 1907986

26 Liu Z, Wang Y, Ren Y, et al. Poly(ionic liquid) hydrogel-based anti-freezing ionic skin for a soft robotic gripper. Mater Horiz, 2020, 7: 919-927

27 Zhang XF, Ma X, Hou T, et al. Inorganic salt induced thermal reversible and anti-freezing cellulose hydrogels. Angew Chem Int Ed, 2019, 58: 7366-7370

28 Lei Z, Wang Q, Sun S, et al. A bioinspired mineral hydrogel as a self-healable, mechanically adaptable ionic skin for highly sensitive pressure sensing. Adv Mater, 2017, 29: 1700321

$29 \mathrm{Hu} \mathrm{M}, \mathrm{Gu} \mathrm{X}, \mathrm{Hu} \mathrm{Y}$, et al. Low chemically cross-linked PAM/C-dot hydrogel with robustness and superstretchability in both as-prepared and swelling equilibrium states. Macromolecules, 2016, 49: 3174-3183

30 Chen X. Making electrodes stretchable. Small Methods, 2017, 1: 1600029

31 Shao C, Meng L, Wang M, et al. Mimicking dynamic adhesiveness and strain-stiffening behavior of biological tissues in tough and self-healable cellulose nanocomposite hydrogels. ACS Appl Mater Interfaces, 2019, 11: 5885-5895

$32 \mathrm{Xu} \mathrm{J}$, Jin R, Duan L, et al. Tough, adhesive and conductive polysaccharide hydrogels mediated by ferric solution. Carbohydr Polym, 2019, 211: 1-10

33 Gao Y, Jia F, Gao G. Transparent and conductive amino acidtackified hydrogels as wearable strain sensors. Chem Eng J, 2019, 375: 121915

34 Han L, Yan L, Wang M, et al. Transparent, adhesive, and conductive hydrogel for soft bioelectronics based on light-transmitting polydopamine-doped polypyrrole nanofibrils. Chem Mater, 2018, 30: $5561-5572$

35 Wang Z, Chen J, Wang L, et al. Flexible and wearable strain sensors based on tough and self-adhesive ion conducting hydrogels. J Mater Chem B, 2019, 7: 24-29

36 Ye Y, Zhang Y, Chen Y, et al. Cellulose nanofibrils enhanced, strong, stretchable, freezing-tolerant ionic conductive organohydrogel for multi-functional sensors. Adv Funct Mater, 2020, 30: 2003430 
Xu J, Jing R, Ren X, et al. Fish-inspired anti-icing hydrogel sensors with low-temperature adhesion and toughness. J Mater Chem A, 2020, 8: 9373-9381

38 Li J, Wang L, Wang X, et al. Highly conductive PVA/Ag coating by aqueous in situ reduction and its stretchable structure for strain sensor. ACS Appl Mater Interfaces, 2020, 12: 1427-1435

39 Lu L, Zhou Y, Pan J, et al. Design of helically double-leveled gaps for stretchable fiber strain sensor with ultralow detection limit, broad sensing range, and high repeatability. ACS Appl Mater Interfaces, 2019, 11: 4345-4352

$40 \mathrm{Wu}$ J, Wu Z, Lu X, et al. Ultrastretchable and stable strain sensors based on antifreezing and self-healing ionic organohydrogels for human motion monitoring. ACS Appl Mater Interfaces, 2019, 11: 9405-9414

41 Xia S, Song S, Li Y, et al. Highly sensitive and wearable gel-based sensors with a dynamic physically cross-linked structure for strainstimulus detection over a wide temperature range. J Mater Chem C, 2019, 7: 11303-11314

42 Huang $\mathrm{H}$, Han L, Fu X, et al. Multiple stimuli responsive and identifiable zwitterionic ionic conductive hydrogel for bionic electronic skin. Adv Electron Mater, 2020, 6: 2000239

43 Hu C, Zhang Y, Wang X, et al. Stable, strain-sensitive conductive hydrogel with antifreezing capability, remoldability, and reusability. ACS Appl Mater Interfaces, 2018, 10: 44000-44010

44 Wang Z, Zhou H, Lai J, et al. Extremely stretchable and electrically conductive hydrogels with dually synergistic networks for wearable strain sensors. J Mater Chem C, 2018, 6: 9200-9207

45 Low ZWK, Li Z, Owh C, et al. Using artificial skin devices as skin replacements: Insights into superficial treatment. Small, 2019, 15: 1805453

46 Morelle XP, Illeperuma WR, Tian K, et al. Highly stretchable and tough hydrogels below water freezing temperature. Adv Mater, 2018, 30: 1801541

47 Bai Y, Chen B, Xiang F, et al. Transparent hydrogel with enhanced water retention capacity by introducing highly hydratable salt. Appl Phys Lett, 2014, 105: 151903

48 Chen H, Gao Y, Ren X, et al. Alginate fiber toughened gels similar to skin intelligence as ionic sensors. Carbohydrate Polyms, 2020, 235: 116018

49 Pan X, Wang Q, Guo R, et al. An integrated transparent, UVfiltering organohydrogel sensor via molecular-level ion conductive channels. J Mater Chem A, 2019, 7: 4525-4535

50 Wei Y, Xiang L, Ou H, et al. MXene-based conductive organohydrogels with long-term environmental stability and multifunctionality. Adv Funct Mater, 2020, 30: 2005135

51 Wang S, Li Q, Feng S, et al. A water-retaining, self-healing hydrogel as ionic skin with a highly pressure sensitive properties. J Taiwan Instit Chem Eng, 2019, 104: 318-329

Acknowledgements This work was supported by the National Natural Science Foundation of China (51873024), and the Science and Technology Department of Jilin Province (20200708102YY and 20190201234JC).

Author contributions Gao Y designed and performed the experiments, and wrote the paper; Wang Y analyzed the data and prepared the figures; Xia S and Gao G revised the manuscript. All authors contributed to the general discussion.

Conflict of interest The authors declare that they have no conflict of interest.

Supplementary Information Supporting data are available in the online version of the paper.

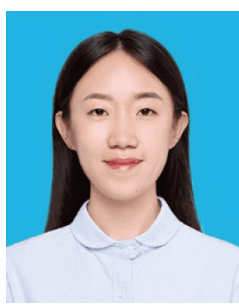

Yang Gao is currently a $\mathrm{PhD}$ candidate at the Polymeric and Soft Materials Laboratory, majored in materials science and engineering at Changchun University of Technology. Her current research interest is focused on the design and synthesis of functional hydrogel materials as well as their applications for wearable sensors and human-machine interfaces.

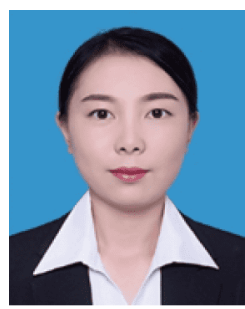

Shan Xia is a lecturer at the School of Chemical Engineering, Changchun University of Technology. She received her $\mathrm{PhD}$ in materials science from Changchun University of Technology in 2020. Dr Xia's current research interests include the design and synthesis of functional polymer hydrogels and their applications in electronic skins, human-machine interfaces, and wearable sensors.

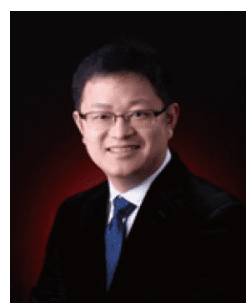

Guanghui Gao is a professor of the School of Chemical Engineering and Advanced Institute of Materials Science of Changchun University of Technology. He obtained his bachelor's degree from Shanghai Jiaotong University and $\mathrm{PhD}$ from Sungkyunkwan University, Korea. He built the Polymeric and Soft Materials Laboratory at Changchun University of Technology. His current research focuses on the application of polymer hydrogels in medical dressings, flexible sensing, and energy storage.

\section{用于人机界面的具有类皮肤性质的环境稳定水凝胶}

高阳, 王元瑞, 夏珊", 高光辉 ${ }^{*}$

摘要 离子水凝胶基传感器已经成为制备可穿戴电子产品的热点. 然而, 皮肤与传感器之间弱的界面粘合力以及水凝胶较差的耐温 性严重影响了传感器的灵敏度和可靠性. 受皮肤结构和功能的启 发, 我们将胶原蛋白整合到化学交联的水凝胶中, 设计出一种新型 的可皮肤附着的且环境稳定的水凝胶. 该水凝胶具有较低的模 量、良好的拉伸性能和良好的弹性. 此外, 胶原蛋白的引入使水凝 胶与包括皮肤在内的多种材料具有牢固的和紧密贴合的界面. 因 此, 该水凝胶具有极高的灵敏度 $(\mathrm{GF}=10.7)$ 、快速响应性、可忽略 的迟滞行为以及广泛的监测范围, 可作为人机界面以采集可靠的 心电图(ECG)信号和辨别各种人体动作. 值得注意的是, 模仿大多 数生物体的耐温机制的水凝胶在零下或常温条件下具有持久的粘 合性、导电性、传感性能和机械稳定性. 这种以皮肤为灵感的策 略为多功能材料的设计提供了一条有效途径, 并有望应用于新一 代电子产品中. 